\title{
Application of wavelet packet in the MIMO-COFDM systems.
}

\author{
Bouamama Réda Sadouki ${ }^{1}$, Mouhamed Djebbouri ${ }^{2}$ \\ ${ }^{I}$ (Electronics Department, University of Sidi Bel Abbès Telecom Laboratory LTTNS , ALGERIA, \\ ${ }^{2}$ (Electronics Department, Professor, University of Sidi Bel Abbès Telecom Laboratory LTTNS , ALGERIA,
}

\begin{abstract}
The insertion of Wavelets, the error-correcting coding and diversity of space multiplexing orthogonal frequency division (OFDM) to improve the performance of wireless systems requires a mastery of all the techniques that are news and find many applications in telecommunications systems. OFDM despite its sulfur benefits many problems (PARP , CFO and ISI) which remains to be solved in a more effective way to increase throughput and reduce energy consumption and improve spectral efficiency, we must add it space diversity ( multiple antennas at the transmitter and receiver), and an error correction coding. The use of wavelets in this communication system can improve its performance in more perspective spectral efficiency (remove the guard interval).

This work aims to study as a pathway based on the use of a MIMO system (multiple antennas at the transmitter and receiver), to reject high power jammers and multipath. The joint use of OFDM and MIMO allows a priori performance improvements and reduce the effect of interference. The integration of the wavelet packet in the OFDM party may make the system less sensitive to interference which is an interesting and desirable benefit that remains to be verified by computer simulations
\end{abstract}

Keywords: FFT-COFDM, DWPT (Discrete Wavelet Packet transform), MIMO-OFDM, MIMO-COFDM.

\section{Introduction}

This article is based on MIMO COFDM system with deferent types use FFT-COFDM, and DWPT-COFDM. MIMO technology has achieved channel capacities and high performance for wireless communications. Although the range of applications of MIMO technique is off several lines of research have developed around multi-antenna systems [1-2]. This study covers various aspects, from the system model and the propagation channel to advanced polarization techniques. Thus, it could be further improved by combining it with some techniques with two types Coded Orthogonal Frequency Division Multiplexing (MIMO-COFDM FFT) and the other with Wavelet Packet transforms Discreet based multicarrier coded modulation (MIMO- DWPT COFDM). COFDM system is a coded multicarrier modulation technique [3], of subdividing the entire signal frequency band into $N$ sub with overlapping frequency channels. Each sub channel is modulated by a separate symbol and the $N$ sub-channels are frequency multiplexed. A frequency guard band is inserted between channels to avoid spectral overlap and eliminate inter symbol interference (ISI) to reduce the effects caused by multipath propagation [4]. The processing here is performed in the frequency domain to the blocks - IFFT / FFT, OFDM allows the user to obtain better spectral efficiency due to the orthogonality of the carrier frequency and the overlapping channels [5-6]. The DWPT-COFDM system is coded multicarrier modulation technique of, and a promising new alternative technique for OFDM established. The treatment here is carried out in the Wavelet Packet transforms Discreet Modulation domain using the blocks - IDWPT / DWPT and However DWPT COFDM, its Wavelet Packet basis functions are finite in time. The inter-symbol orthogonality in Wavelet Packet transform is maintained due to the passage of orthogonal waveforms. In the Wavelet Packet et transform [7-8]. The symbols are overlapped in the time domain. This feature increases the symbol duration that provides tolerance, but also prevents the availability of CP [9]. In this research, we provide a detailed report on the very significant differences between systems FFT-COFDM and DWPT-COFDM based on MIMO. And this technique is the greatest motivation for continuing MIMO-FFT COFDM systems from the levels of performance degradation due to inter-symbol interference (ISI). An effective technique to mitigate this degradation is to introduce CP, a previous signal dual-partition that can be added to each block COFDM. CP well designed will significantly increase tolerance. However, DWPT -COFDM, its wavelet basis functions are finite in time [10][11].

\section{Model Conventional MIMO-COFDM System: FFT-COFDM}

MIMO-COFDM MIMO-COFDM can be used to simulate the effect of the ISI that the length of a guard interval CP varies. It considers the BER performance of an OFDM system with 64 points and 16 carrier $\left(v_{c}=\mathrm{N}\right.$ $N$ sub of 16-QAM in the channel or allowing AWGN results allowing a multipath Rayleigh, it is clear that the $B E R$ performance with CP of length 16 samples is compatible with that of the result it is also clear that the BER performance in a channel for allowing AWGN results is consistent with the analytical results. This is true 
regardless of the long GI, because there is no delay in authorizing the multipath allowing $A W G N$ results pui what each sub channel is modulated by a separate symbol and the $N$ sub-channels are multiplexed in frequency (10). A band of custody is inserted between the frequency channels in order to avoid overlapping spectral and eliminate the inter-symbol interference (ISI). This technique separates the channels high speed in a plurality of lower fares parallel. The serial-to-parallel converter after the quadrature modulator therefore generates vectors write under the following formula:

$\overline{\mathrm{d}}_{1}=\left[\begin{array}{c}\mathrm{d}_{\mathrm{i}}(0) \\ \mathrm{d}_{\mathrm{i}}(1) \\ \mathrm{d}_{\mathrm{i}}(2) \\ \cdots \\ \mathrm{d}_{\mathrm{i}}(\mathrm{n}) \\ \cdots \\ \mathrm{d}_{\mathrm{i}}(\mathrm{N}-1)\end{array}\right], \mathrm{i}=0,1,2$

and

$\mathrm{d}_{\mathrm{i}}(\mathrm{n})=\mathrm{a}_{\mathrm{i}}(\mathrm{n})+\mathrm{j} \mathrm{b}_{\mathrm{i}}(\mathrm{n}) ; \mathrm{i}=1,2,3, \ldots, \mathrm{N}-1$

Consider one of the vectors $\overline{\mathrm{d}}_{1}$ given by equation (1.1) and (1.2). This vector contains $\mathrm{N}$ digital symbols which make up the OFDM symbol. Apply to $\overline{\mathrm{d}}_{1}$ the inverse discrete Fourier transform which generates the vector $\overline{\mathrm{D}}_{1}$ is expressed as follows:

$\overline{\mathrm{D}_{1}}(\mathrm{~K})=\frac{1}{\mathrm{~N}} \sum_{\mathrm{n}=0}^{\mathrm{N}-1} \mathrm{~d}_{\mathrm{i}}(\mathrm{n}) \mathrm{e}^{\frac{2 \pi}{\mathrm{N}} \mathrm{kn}} ; \mathrm{k}=0,1,2,3, . ., \mathrm{N}-1$

Transforming the $\mathrm{N}$ symbols in the time series Vector $\mathrm{D}_{\mathrm{i}}$ whose elements are separated by a time interval $\mathrm{T}_{\mathrm{U}}$ we obtain:

$\mathrm{D}_{\mathrm{i}}(\mathrm{K})=\frac{1}{\mathrm{~N}} \sum_{\mathrm{n}=0}^{\mathrm{N}-1} \mathrm{~d}_{\mathrm{i}}(\mathrm{n}) \mathrm{e}^{\mathrm{j} \frac{2 \pi}{\mathrm{N}} \mathrm{kn}} \Leftrightarrow \mathrm{D}_{\mathrm{i}}\left(\mathrm{KT}_{\mathrm{U}}\right)=\frac{1}{\mathrm{~N}} \sum_{\mathrm{n}=0}^{\mathrm{N}-1} \mathrm{~d}_{\mathrm{i}}\left(\mathrm{nT}_{\mathrm{U}}\right) \mathrm{e}^{\mathrm{j} \frac{\mathrm{nT}}{\mathrm{N}} \mathrm{kT} \mathrm{Un}}$

$\mathrm{D}_{\mathrm{i}}(\mathrm{K})=$

$\frac{1}{\mathrm{~N}} \sum_{\mathrm{n}=0}^{\mathrm{N}-1}\left[\mathrm{a}_{\mathrm{i}}(\mathrm{n}) \cos \left(\frac{2 \pi}{\mathrm{N}} \mathrm{nk}\right)-\mathrm{b}_{\mathrm{i}}(\mathrm{n}) \sin \left(\frac{2 \pi}{\mathrm{N}} \mathrm{nk}\right)\right]+\mathrm{j}\left[\frac{1}{\mathrm{~N}} \sum_{\mathrm{n}=0}^{\mathrm{N}-1}\left(\mathrm{~b}_{\mathrm{i}}(\mathrm{n}) \cos \left(\frac{2 \pi}{\mathrm{N}} \mathrm{nk}\right)+\mathrm{a}_{\mathrm{i}}(\mathrm{n}) \sin \left(\frac{2 \pi}{\mathrm{N}} \mathrm{nk}\right)\right)\right]$

If we ask $\frac{f_{n}}{N_{U}}+\frac{N}{T_{S}}$ represents the frequency spacing between sub $\mathrm{NT}_{U}, T_{\mathrm{a}}$ carriers, $t_{U}=\mathrm{KT}_{U}$ and $T_{U}$ where is the duration of a digital symbol sequence $d_{n}$ serial data, then we get:

$D_{i}\left(t_{k}\right)=I_{i}\left(t_{k}\right)+J Q_{i}\left(t_{k}\right)$

From equation (1.2) and (1.6) one has:

$$
\begin{aligned}
& \left.\mathrm{I}_{\mathrm{i}}\left(\mathrm{t}_{\mathrm{k}}\right)=\frac{1}{\mathrm{~N}} \sum_{\mathrm{n}=0}^{\mathrm{N}-1}\left|\mathrm{~d}_{\mathrm{i}}(\mathrm{n})\right| \cos \left(2 \pi \mathrm{f}_{\mathrm{n}} \mathrm{t}_{\mathrm{k}}\right)+\varphi(\mathrm{n})\right] \\
& \left.\mathrm{Q}_{\mathrm{i}}\left(\mathrm{t}_{\mathrm{k}}\right)=\frac{1}{\mathrm{~N}} \frac{1}{\mathrm{~N}} \sum_{\mathrm{n}=0}^{\mathrm{N}-1}\left|\mathrm{~d}_{\mathrm{i}}(\mathrm{n})\right| \sin \left(2 \pi \mathrm{f}_{\mathrm{n}} \mathrm{t}_{\mathrm{k}}\right)+\varphi(\mathrm{n})\right]
\end{aligned}
$$

(1.1), (1.1), (1. 2), (1. 3), (1. 4), (1. 5), (1. 6), (1. 7) formula is given by [12]-[13]-[14]-[15]-[16].

The OFDM signal, $s(t)$ consists of the real part, $s(t)=I_{i}(t)$. In the presence of the inter symbol interference caused by the transmission channel, the orthogonality properties between the subcarriers are not kept. One way to circumvent the problem of selectivity is indefinitely increase the number of subcarriersN. However, this solution is limited by the temporal coherence of the channel [15]. This 
guard interval must be longer than that of the impulse response I'étendue to absorb the effect multi channel paths. Therefore, the useful symbol duration is free of intersymbol interference symbols and the orthogonality condition is maintained. We therefore inserted between two transmitted a time interval called time grade $\mathrm{T}_{0}$ symbols. The purpose of the guard interval is explained by the following. The symbols in the passing channel multipath echoes and undergo a transmitted symbol can be received by the receiver as a plurality of attenuated and delayed symbols. A symbol transmitted at time $\mathrm{n}_{\mathrm{T}}$ can overlap an echo from the transmitted symbol at time $(\mathrm{n}-1) \mathrm{T}_{\mathrm{g}}$. The total duration of the transmitted symbol is then $\mathrm{T}=\mathrm{T}_{\mathrm{a}}+\mathrm{T}_{\mathrm{G}} \bullet$ to prevent interference, it is necessary to choose a larger guard time that the delay spread of the channel where $\mathrm{T}_{\mathrm{G}}>\tau_{\max }$. [16].

\section{Discrete Wavelet Packets Transform (Dwpt)}

The packets of wavelet transforms used for data compression of it functions are localized in the two areas (time / frequency). The parameter of a packet of wavelets starts of a base pair of mirror filters in quadrature $g$ and $h$ that meets the following conditions [17] .the logarithmic division of bandwidth in the transformed into wavelet is not well suited for multiporteuses communication suchas COFDM systems MIMO [18]. Packet of wavelet transforms are generalization of the wavelet transform or the orthogonal basis functions are 'packets of wavlets", as we have already the researcher discussed, in process DWPT, each level is calculated only in passing the old approximation of coefficients by low-pass and high-pass filters [ 17].consequently DWPT have more flexibility or the bandwidth can be arbitrarily divided according to the pruning of trees, and the functions of packets of wavelets, $\zeta_{j, k}(t)$ given by [19].

$\zeta_{j, k}(t)=2^{i} \zeta^{n}\left(2^{j} t-k\right) \quad j, k \in Z$

Where $\zeta^{\mathrm{n}}$ (no subscripts) is to have $=k=0$. The extra index $n=0$ is called the modulation parameter or oscillation parameter. The first two wavelet packet functions are known as the usual scaling function and wavelet function

$$
\begin{aligned}
& \varphi(t)=\sum_{h=-\infty}^{\infty} h_{n} \sqrt{2} \varphi(2 t-n), n \in Z \\
& \psi(t)=\sum_{h=-\infty}^{\infty} g(n) \sqrt{2} \varphi(2 t-n), n \in Z \\
& \zeta^{2 n+1}=\sum_{k=-\infty}^{\infty} g(n) \sqrt{2} \zeta_{n}(2 t-k), k \in Z \\
& c_{j}(k)=\sum_{m=-\infty}^{\infty} h(m-2 k) c_{j+1}(m) \\
& d_{j}(k)=\sum_{m=-\infty}^{\infty} g(m-2 k) c_{j+1}(m)
\end{aligned}
$$

The coefficients can be computed via

$$
c_{j}^{n}(k)=\int_{-\infty}^{+\infty} w_{j k}^{n}(t) d t
$$

Combining with Eqs. (1.13, 1.14) And (1.15), we can obtain the following relationship

$$
\begin{aligned}
& c_{j}^{2 n}(k)=\sum_{m=-\infty}^{\infty} h(m-2 k) c_{j+1}^{n}(m) \\
& c_{j}^{2 n+1}(k)=\sum_{m=-\infty}^{\infty} g(m-2 k) c_{j+1}^{n}(m)
\end{aligned}
$$

(1.10), (1.11), (1.12), (1.13), (1.14), (1.15), (1.16), (1.17) formula is given by [17]-[18]-[19]-[20].

And this is equivalent to j-level full wavelet packet decomposition (full binary tree structure). For OFDM systems that require uniformly division of bandwidth, a DWPT with a full binary tree structure is utilized (see Fig1.1).

The inverse discrete wavelet packet transform (IDWPT), also called the wavelet packet reconstruction (WPR), has a 'mirror image' process of the DWPT with a similar tree structure, where the dataflow are from 'leaves' to 
the 'root', as shown in Fig (1.1) the 'leaves' furthest to the left are the packet coefficients, followed by convolution with reconstruction high-pass filter $h$ ' (or low-pass filter $g$ '). The high-and low-pass branches are then summed up generating a new sequence. After $n$ levels of such iterative processes, the 'root' furthest to the right gives the time-domain transformed data. For data transmission, DWPT and IDWPT must be used as a pair with the reconstruction and decomposition filters having the following relationship [20]-[21].

Where $g(n)$ is called the wavelet function coefficients (or wavelet filter). The relationship between these two coefficients is restricted by the orthogonality condition, given by [22]:

$g(n)=(-1)^{n} h(N-1-n) \quad$ And $\quad h(n)=\frac{1}{\sqrt{2}}(-1,1), g(n)=\frac{1}{\sqrt{2}}(1,1)$

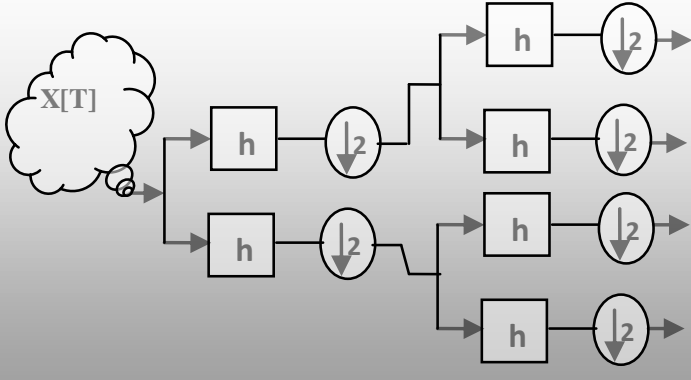

a) Discrete wavelet packet transforms (DWPT)

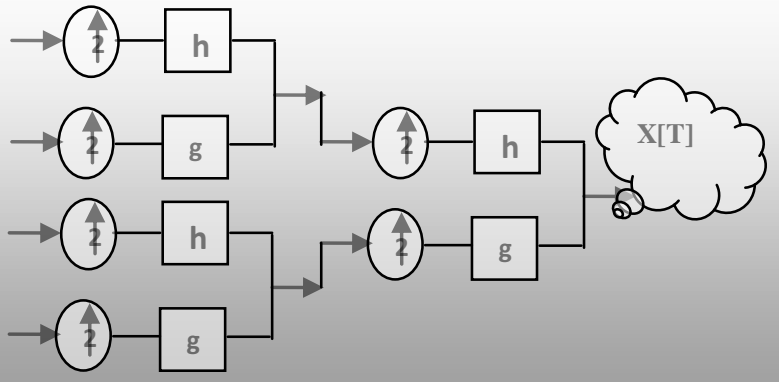

(b) Inverse discrete Wavelet basket transforms (IDWPT)

IV. Simulation And Results

FIG 1.1: (a) (b) reconstruction and decomposition filters

We have carried out numerical simulations to compare the performance (BER) between two systems MIMO DWPT-COFDM and MIMO FFT-COFDM. The simulation parameters are shown in the table (1) and figure (1.1). The two DWPT FFT-COFDM and-COFDM systems are developed, analyzed, and simulated in Matlab version 7. The results of the performance of the channel system, and selective channel allowing AWGN results are obtained by using the COFDM parameters as indicated in the table (1.1):

Table (1.1) Simulation Parameters

\begin{tabular}{|l|l|l|}
\hline Parameter & FT-COFDM & WPT-COFDM \\
\hline Modulation & $\begin{array}{l}\text { BPSK,QPSK, } \\
\text { 16-QAM }\end{array}$ & $\begin{array}{l}\text { BPSK,QPSK, } \\
\text { 16-QAM }\end{array}$ \\
\hline No. of sub-carriers & 32 & 32 \\
\hline $\begin{array}{l}\text { No. of bits per } \\
\text { symbol }\end{array}$ & 32 & 32 \\
\hline No. of FFT points & $16,32,64,128$ & $16,32,64,128$ \\
\hline Data Rate & $112 \mathrm{Mbps}$ & $112 \mathrm{Mbps}$ \\
\hline Wavelet & - & Haar \\
\hline Channel Model & AWGN & AWGN \\
\hline antenna(Mt,Mr) & $(1,1) ;(2,2)$ & $(1,1) ;(2,2)$ \\
\hline Code rate & $1 / 2$ & $1 / 2$ \\
\hline CP & $1 / 8$ & - \\
\hline
\end{tabular}

\section{BER Performance In MIMO DWPT-COFDM and MIMO FFT-COFDM At The AWGN} Channel

Represents the performance MIMO DWPDT-COFDM with l QAM of different points of 16, 32 and 64 on the channel allowing AWGN results. The results are presented in the obtained more performance improves (gain is wide between the systems which use 8 points to the QAM system with 16, 32, 64 points for SNR values higher, when the SNR increases. 


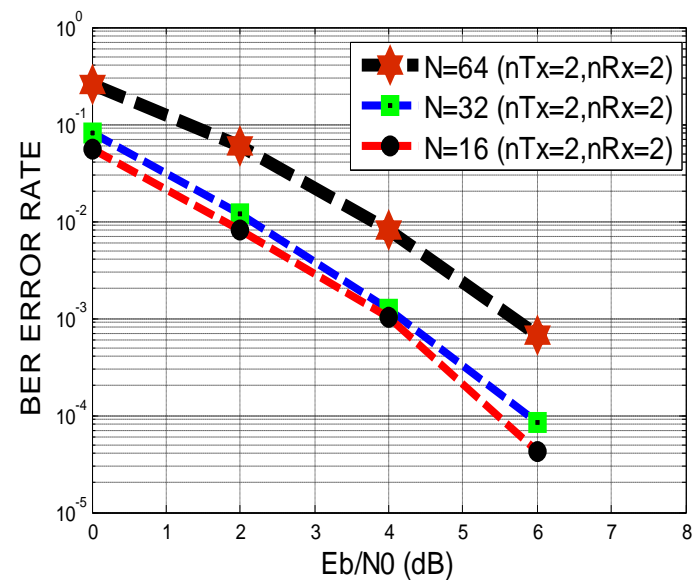

FIG 1.2 presented performance for different values of $\mathrm{N}$, the number of sub-carriers on DWPT-COFDM Figure 1.2 shows results for the results for the FFT-COFDM with QAM 16, 32 and 64 on allowing AWGN results. The performance is wide between systems that use 8 points to the QAM system with 64 points for SNR values higher. The figure (1.4) presents the results for different values of $N$, the number of sub-carriers on MIMO FFT-COFDM. It is observed, increase the number of subcarrier decrease the width of each band of sub-carriers, so that for less than the width of the band of coherence which has improved the performance of system DWPT-COFDM last mesh is compared FFT-COFDM.

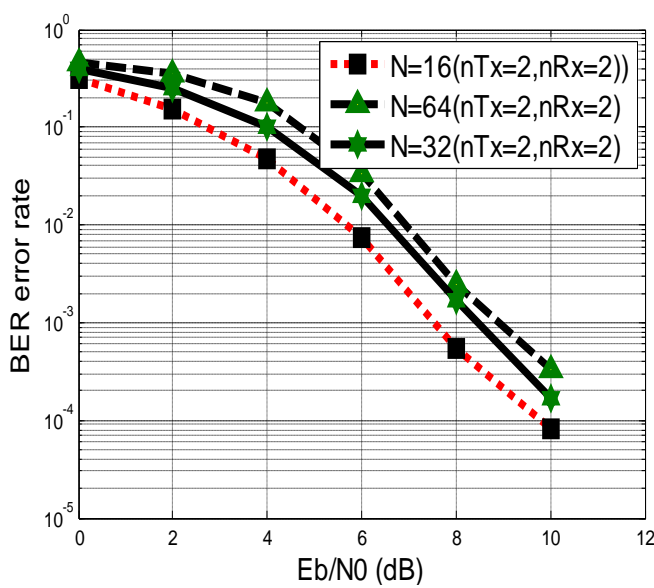

FIG 1.3presented performance for different values of $\mathrm{N}$, the number of sub-carriers on MIMO-COFDM

The figure 1.3 presented performance for different values of $N$, the number of sub-carriers on MIMO FFT-COFDM and with an identical recess bandwidth. It is observed, increase the number of subcarrier decrease the width of each subcarrier band, so that for less than the width of the coherence band which improved the performance of system DWPT -COFDM last mesh is compared FFT-COFDM.

Higher performance gains are also observed when the SNR increases. 


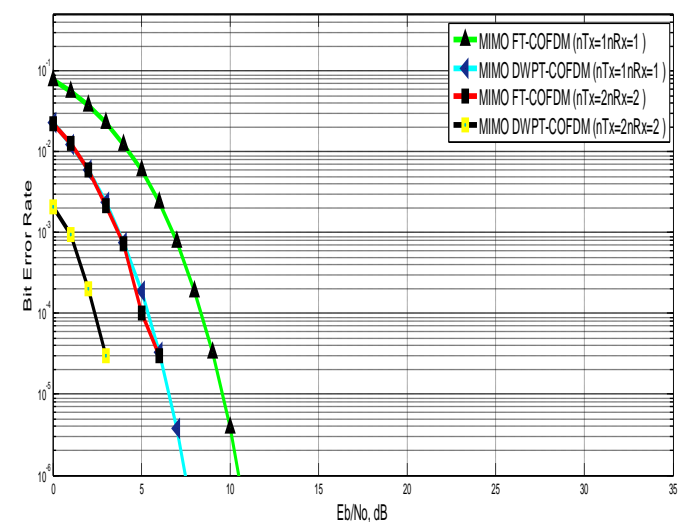

Figure 1.4: BER performance MIMO between FFT-OFDM and DWPT-OFDM in AWGN, for different values of $n T_{x}, n_{x}$.

This simulation we calculate the bit error rate as a function of Signal to Noise for different SNR The figure (1.4) we calculate the bit error rate for different SNR we have a bit error rate to decrease by increasing the number of antennas to the front desk and also reduces the number of symbol, and it will transmit to a bit error rate to decrease more in the case of a DWPT MIMO-COFDM and best by what this figure clearly demonstrates that the performance of the OFDM system DWPT is better than the FFT-OFDM system.

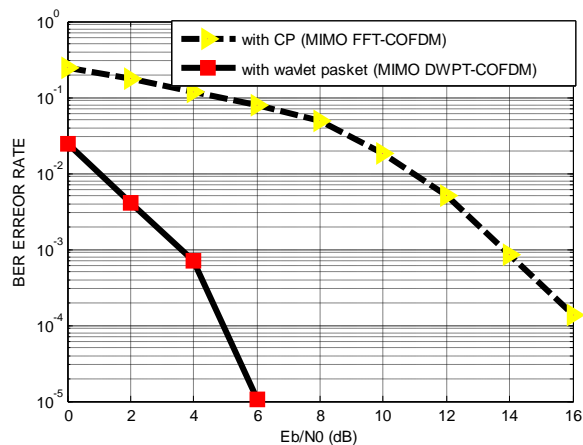

Figure 1.5: BER performance MIMO between FFT-OFDM and DWPT-OFDM in AWGN for with CP, and DWPT

The figure (1.5) shows the evolution for using the cyclic prefix on MIMO FFT-COFDM or not on MIMO DWPT-COFDM. A saturation effect is observed: when the prefix length exceeds the length of the impulse response, there is no of the ISI and further increase is unnecessary and even harmful in the sense that the prefix consumes a part of the bandwidth. It may be noted that the saturation begins before 800ns, but comparatively DWPT-COFDM is the best performance with wavelet packet that we have seen the absence of IES and with the cyclic prefix is larger than the delay spread, the same time there has been a lack of small IES.

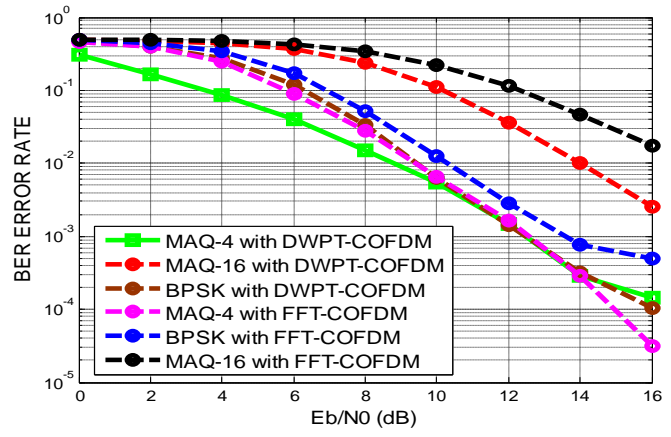

Figure 1.6: BER performance MIMO between FFT-OFDM and DWPT-OFDM in AWGN of different constellation 
Figure 1.6, shows performance degradation when the number of states of the constellation used increases. This results from the decrease of the Euclidian distance between the constellations for different states of increasing size, except for the passage of a BPSK constellation in a constellation for QPSK in this case, the distances are identical (exploiting only the BPSK while the actual constellations QPSK (or more) part also exploiting the imaginary part).

\section{Conclusions}

In this paper, we proposed a model MIMO-DWPT COFDM of the simulation to test and compared it with MIMO-FFT COFDM. After the results have approved the proposed and provided the concept design simulation is yielding positive results and be seen. The bit error rate performance in DWPT based MIMO COFDM with BPSK, QPSK, 16-QAM modulation scheme is more efficient than FFT based MIMO COFDM. By applying the adaptive modulation method in MIMO-DWPT COFDM, the simulation results of bit error rate to signal to noise ratio gives a better BER performance comparing to the MIMO-DWPT COFDM and MIMO-FFT COFDM with BPSK, QPSK in AWGN channel. From the simulation results we obtained it can be concluded that the application of different uses either Wavelet Packet where FFT on MIMO-COFDM systems gives performance refers dependent on their structures. As the number of antennas is big plus performance is good. By cons between two systems with the same variety, best performance is obtained from the system with the total number of the largest antennas Wavelet Packet.

\section{References}

[1] D. Gesbert and J. Akhtar. Breaking the barriers of Shannon's capacity: An overview of MIMO wireless system. Telektronikk Telenor Journal, January 2002.

[2]Weinston, S. B., \& Ebert, P. M. (1971). Data transmission by frequency division multiplexing using the discrete Fourier transform. IEEE Trans., COM-19(5), 628-634.

[3] B. Le Floch, M. Alard, and C. Berrou. Coded orthogonal frequency division multiplex. IEEE Proceedings, 83(6):982-996, 1995.

[4] R. Van Nee and R.Prasad,“OFDM for Wireless Multimedia Communications”, Artech House Publishers, 2000.

[5] Weinstein, S. and Ebert, P.; "Data Transmission by Frequency-Division Multiplexing Using the Discrete Fourier Transform" IEEE Trans. on Commun. vol. 19 Issue: 5, pp. $628-634$, Oct.1971

[6] L.J. Cimini, "Analysis and simulation of a digital mobile channel using orthogonal frequency division multiplexing", IEEE Trans. Commun., vol. COM-33, pp. 665-675. July 1985

[7] R. Coifman, Y. Meyer, S. Quake and M. V. Wickerhauser, "Signal processing and compression with wavelet packets", NATO ASI Series C Mathematical and Physical Sciences, vol. 442, pp. 363, (1994)

[8] R. Coifman and Y. Meyer, "Orthonormal Wave Packet Bases," Technical Report, Dept. of Math., Yale Univ., (1990).

[9] Weinston, S. B., \& Ebert, P. M. (1971). Data transmission by frequency division multiplexing using the discrete Fourier transform. IEEE Trans., COM-19(5), 628-634.

[10] Y.Meyer, Ondelettes, algorithmes, Ed. Hermann, Paris 1990.

[11] Z. Baharav and Y. Leviatan . "Impedance Matrix Compression (IMC) Using Iteratively Selected Wavelet Basis . " IEEE Trans. Antennas Propagat. 46( 1997 ), 226 - 233

[12]Webb, W., \& Hanzo, L. (1994). Modem quadrature amplitude modulation: princip/es and applications for fixed and wireless communications. London, Angleterre: Pentech Press.

[13]Weinston, S. B., \& Ebert, P. M. (1971). Data transmission by frequency division multiplexing using the discrete Fourier transform. IEEE Trans., COM-19(5), 628-634.

[14]Xiong, F. (2000). Digital modulation techniques. Boston, Mass.: Artech House.

[15]Ôberg, T. (2001). Modulation, detection and coding. Chichester, Angleterre: J. Wiley and Sons.

[16] G. Donovan, J. Geronimo, D. Hardin, P. Massopust, «Construction of orthogonal wavelets using fractal interpolation functions», SIAM J. Math. Anal., vol. 27, N॰4,pp. 1158-1192, 1996

[17] Cherubini, G. (2000) Filter bank modulation techniques for very high-speed digital subscriber lines.IEEE Commun. Mag., 38(5), 98104

[18] N. Erdol, F. Bao, and Z. Chen, "Wavelet modulation: a prototype for digital communication systems," in IEEE Southcon Conference, pp. 168-171 (1995). 
[19] [N. Erdol, F. Bao, and Z. Chen, "Wavelet modulation: a prototype for digital communication systems," in IEEE Southcon Conference, pp. 168-171 (1995).

[20] S. G. Mallat, "Theory for Multiresolution Signal Decomposition: The Wavelet Representation", IEEE transactions on pattern analysis and machine intelligence. vol. II, no. 7, pp. 674-693 (1989).

[21] G. Donovan, J. Geronimo, D. Hardin, P. Massopust, «Construction of orthogonal wavelets using fractal interpolation functions», SIAM J. Math. Anal., vol. 27, №4,pp. 1158-1192, 1996

[22] R. Coifman and Y. Meyer, "Orthonormal Wave Packet Bases," Technical Report, Dept. of Math., Yale Univ., (1990). 